\title{
Comparative Seroprevalence of Hepatitis B Virus among in-Mates and Low Risk Voluntary Blood Donors in Garissa, Kenya
}

\author{
Vincent Bahati1,2, Wallace Bulimo ${ }^{3}$, George Gachara² \\ ${ }^{1}$ Directorate of Health, Department of Medical Laboratory Sciences, Garissa County, Kenya \\ ${ }^{2}$ Department of Medical Laboratory Science, Kenyatta University, Nairobi, Kenya \\ ${ }^{3}$ Department of Biochemistry, University of Nairobi, Nairobi, Kenya \\ Email: vinniebaha@yahoo.com
}

How to cite this paper: Bahati, V., Bulimo, W. and Gachara, G. (2021) Comparative Seroprevalence of Hepatitis B Virus among in-Mates and Low Risk Voluntary Blood Donors in Garissa, Kenya. Journal of Biosciences and Medicines, 9, 85-95. https://doi.org/10.4236/jbm.2021.97010

Received: May 25, 2021

Accepted: July 12, 2021

Published: July 15, 2021

Copyright $\odot 2021$ by author(s) and Scientific Research Publishing Inc. This work is licensed under the Creative Commons Attribution International License (CC BY 4.0).

http://creativecommons.org/licenses/by/4.0/

\begin{abstract}
Hepatitis B virus (HBV) infection is a potentially life-threatening infection that attacks the liver and can cause both acute and chronic disease. This creates a high risk of death from cirrhosis and liver cancer. Hepatitis B infection poses a major health concern globally. It is estimated that 257 million people are infected globally with 780,000 deaths reported annually. In Kenya, HBV prevalence stands at chronic states of intermediate range (5\% - 7\%) and high ( $\geq 8 \%$ ) with regional variations. Garissa County carries a high HBV infection risk with a reported prevalence of $14.1 \%$ in pregnant women attending antenatal care (ANC) clinics. This study was carried out to determine and compare the seroprevalence of HBV among in-mates and voluntary blood donors at Garissa Main Prison and Garissa County referral hospital respectively in Garissa, Kenya. A total of 130 in-mates and 130 voluntary blood donors were sampled in this study. Serum was tested for Hepatitis B surface antigen (HBsAg) using a rapid test cassette (Amitech Diagnostics Inc.). A questionnaire was also used to collect socio-demographic factors of the study participants. Data were entered and analyzed using SPSS version 20. Majority of the study participants were males (86.9\% among inmates and $95.4 \%$ among blood donors). Majority (76.2\%) of the in-mates and of the donors (83.1\%) were aged between 20 - 40 years while majority $(51.4 \%$ of the donors and $81.5 \%$ of in mates) had only a primary school level of education. HBV seroprevalence was significantly higher among in mates compared to blood donors. Out of the total number of in-mates tested, 7 (5.4\%) were HBV seropositive. Conversely, among blood donors 4 (3.1\%) were seropositive. There was a significant association between HBV seropositivity and gender among both the blood donors and in-mates. There was no significant association between HBV seropositivity and both level of education and age. No data currently
\end{abstract}


exists on HBV seroprevalence in Kenyan prisons and these study findings may be used as a proxy for other prisons within the country. Further studies to determine other predisposing risk factors should be conducted. Additionally, molecular studies to determine circulating HBV genotypes in this group of people and region are required.

\section{Keywords}

WHO-World Health Organization, IDU-Intravenous Drug Use, GCRH-Garissa County Referral Hospital, HBsAg-Hepatitis B Surface Antigen, HBV-Hepatitis B Virus, KNBTS-Kenya National Blood Transfusion Services

\section{Background}

Hepatitis B is a viral infection that is caused by Hepatitis B virus [1]. It is a potentially life threatening infection that attacks the liver and can cause both acute and chronic disease [1]. This creates a high risk of death from cirrhosis and liver cancer [1]. Hepatitis B virus (HBV) infection, poses a major health concern globally. It is estimated that 257 million people are infected globally [2] with 780,000 deaths reported annually [3]. Global chronic HBV infections stand at more than 240 million cases with 100 million HBV affected people living in Africa [4]. Africa has varying HBV prevalence rates of intermediate (2\% - 7\%) to high $\geq 8 \%$ [4]. Hepatitis $B$ infection high endemicity of over $8 \%$ is shown in developing regions with surging populations such as China, South-East Asia, Amazon basin and Sub-Saharan Africa [5]. Chronic pattern states are evident in these regions with majority of them occurring during infancy and portraying asymptomatic states.

Kenya has been categorized with other sub-Saharan African countries with a HBV infection burden of chronic states of intermediate range (2\% - 7\%) and high ( $\geq 8 \%$ ) [4]. Studies have shown HBV infection disparity in Kenya among various study populations, with $5.1 \%$ prevalence in areas near Nairobi among rural settler community, $15.3 \%$ in Turkana County among pregnant women [6], $3.46 \%$ in Kisumu, Homabay and Siaya Counties [7] and 2.3\% in Nairobi [8] among blood donors.

Diagnosis of $\mathrm{HBV}$ is routinely done by measurement of various $\mathrm{HBV}$ specific antigens and antibodies. The most routinely used serological marker is the Hepatitis B virus surface antigen (HBsAg). This is a HBV protein that is found on the virion surface that is the first detectable antigen during $\mathrm{HBV}$ infections. Detection of this antigen is invariably associated with HBV infection. However, it can be absent during early infections [9].

Hepatitis B e antigen ( $\mathrm{HBeAg}$ ) appears shortly after the production of HBsAg. The appearance of this antigen, relates to active replication of the virus [10]. The detection of $\mathrm{HBeAg}$ relates to the infectivity of the patient and also defines acute 
$\mathrm{HBV}$ infection. Hepatitis B core antigen $(\mathrm{HBcAg})$ is used in determination of viral replication. Antibodies to these antigens are also useful in determining the phase of infection.

Transmission of HBV infection is associated with certain risk factors such as blood transfusions, caring for the sick who are infected, working in healthcare settings and living in high-risk prisons. Viral transmission occurs through exposure to body fluids and infectious blood enhanced via intravenous drug use and unprotected sex. Exposure can also occur at the time of birth [11]. Significant risk to transmission, has been identified through tattooing and acupuncture [12].

Prisoners are classified as high risk persons in terms of HBV infection [13]. Prisons contain populations with higher rates of blood borne infections including HBV: people who exchange sex for drugs and those who inject drugs. There is need to address these emerging problems because prisoners who might have been infected with HBV, join the general population after their jail term, which poses a risk of spread of HBV infection to the general population.

There is a reported prevalence of $14.1 \% \mathrm{HBV}$ infections amongst pregnant women attending ANC clinics in Garissa County [14], which represents a significant proportion of the general population. This translates to a high HBV endemicity according to the WHO criteria.

Based on these reports, this study sought to determine and compare the seroprevalence of HBV among the high risk in-mates at the Garissa Main Prison and the low-risk voluntary blood donors in the general population at Garissa County Referral Hospital in Garissa County.

\section{Methodology}

\subsection{Study Area}

The study was conducted at Garissa Main Prison and Garissa County Referral Hospital (GCRH) within Garissa Township area of Garissa County. Garissa County is located in North Eastern region of Kenya. It has six sub Counties; Balambala, Dadaab, Fafi, Garissa, Hulugho, Ijara and Lagdera. It borders Wajir County to the North, Lamu County to the South, Tana River County to the West, Isiolo County to the North West and shares an international boundary with Somalia to the East (Figure 1).

\subsection{Study Design}

This was a cross-sectional study conducted among in-mates at Garissa Main Prison and voluntary blood donors at GCRH.

\subsection{Ethical Considerations and Confidentiality}

The study was approved by the Kenyatta University Ethics and Review Committee (KU-ERC, PKU/2043/I1190). Clearance was also sought from the Trainings Board and Commissioner General for Kenya Prisons Service and the Directorate 


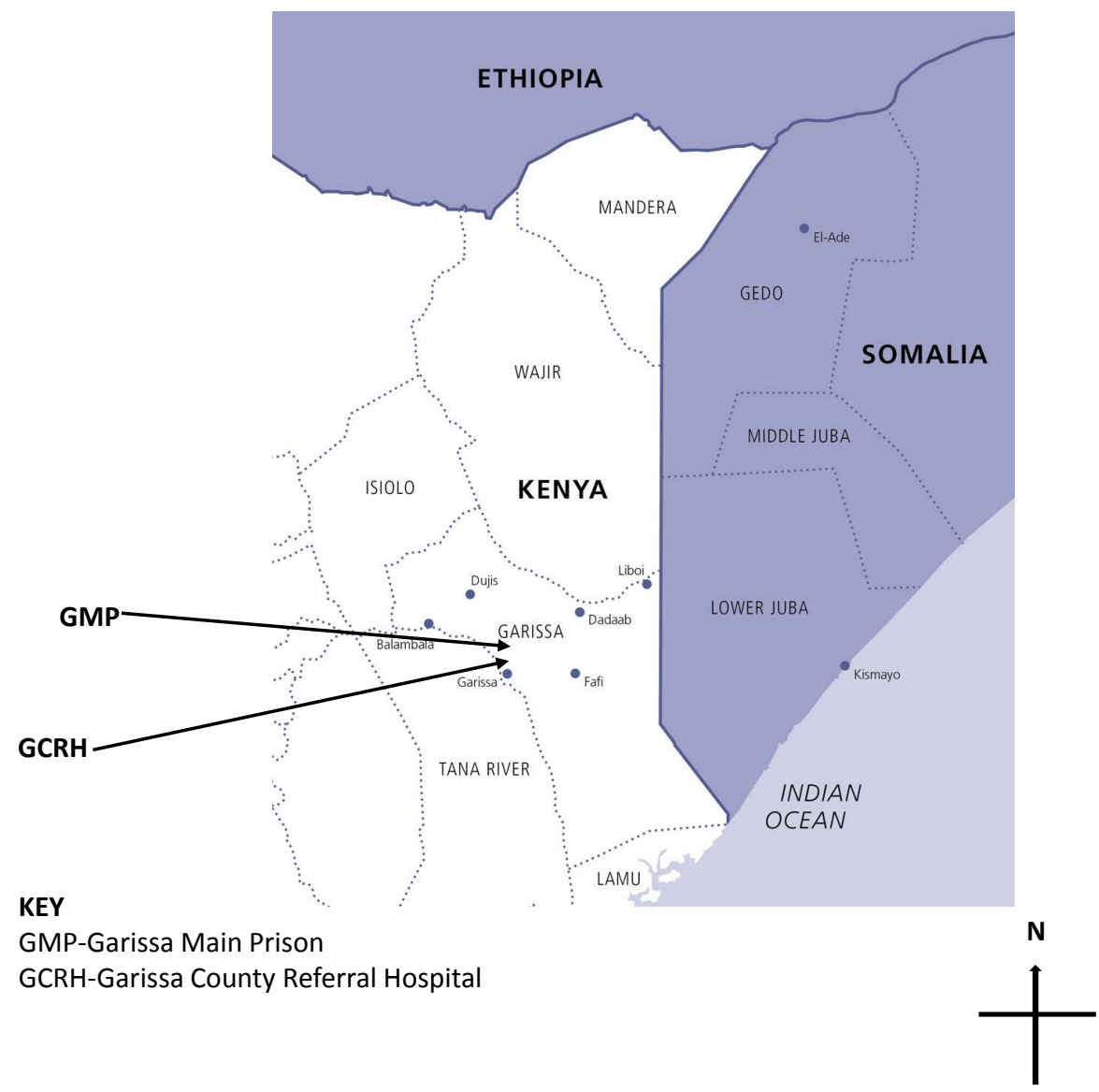

Figure 1. A map showing the study sites.

of Kenya National Blood Transfusion Services (KNBTS). A research permit to conduct the study was obtained from the National Commission for Science, Technology \& Innovation (NACOSTI) under License No. NACOSTI/P/20/4150.

In addition, written informed consent was sought from each participating in-mate and donor and none was remunerated or coerced to participate. The study participants' identity was coded using special numbers to ensure confidentiality.

\subsection{Inclusion Criteria}

Consenting In-mates of all sexes aged 18 years and above were included. However, in-mates who were aged between 16 - 18 years and consented were also considered. Voluntary blood donors of all sexes aged between 18 to 65 years of age and who weighed $50 \mathrm{~kg}$ and who met the KNBTS blood donation criteria were also included.

\subsection{Exclusion Criteria}

In-mates who did not consent and were aged below 16 years of age were excluded. Equally, voluntary blood donors weighing less than $50 \mathrm{~kg}$ and aged below 18 and above 65 years of age were also excluded. 


\subsection{Sample Size Determination}

Minimum sample size for the study was calculated based on a previous formula used for comparing two study populations [15].

$$
n=\frac{\left\{Z_{1-\alpha / 2} \sqrt{2 P(1-P)}+Z_{1-\beta} \sqrt{P_{1}\left(1-P_{1}\right)+P_{2}\left(1-P_{2}\right)}\right\}^{2}}{\left(P_{1}-P_{2}\right)^{2}}
$$

where:

$\alpha=$ Type I error (0.05);

$\beta=$ Type II error $\left(0.20^{a}\right.$ or $\left.0.10^{b}\right)$;

At $95 \%$ confidence, $Z_{1-\alpha / 2}=1.96$.

At $80 \%$ power, $Z_{1-\beta}=0.842^{a}$.

At $90 \%$ power, $Z_{1-\beta}=1.28^{b}$.

$P_{1}=$ Assumed/Estimated Proportion of outcome 1 (prevalence of HBV in prisoners) $=0.50$

$P_{2}=$ Estimated proportion outcome 2 (prevalence of HBV in general population) $=0.30$.

$$
n=\frac{\{1.36+0.87\}^{2}}{(0.50-0.30)^{2}}, \quad n=124 .
$$

2 populations each with 124 , rounding up to 130 . For both populations: $130 \times$ $2=260$.

\subsection{Sampling Technique}

Purposive sampling technique was adopted.

\subsection{Sample Collection and Processing}

Venous blood approximately $4 \mathrm{ml}$ was collected once into plain vacutainer from each consenting adult in-mate and voluntary blood donor after sterilizing thoroughly the puncture site. Initial laboratory procedures were carried out at GCRH laboratories. Serum was prepared by centrifuging blood at $3000 \mathrm{xg}$ for 5 minutes for serological work.

Later, aseptic aspiration of serum was done and aliquoted into sterile labelled cryotubes which were then archived at $-80^{\circ} \mathrm{C}$ for future molecular studies.

\subsection{Serological Testing}

All the serum samples underwent serological testing for hepatitis B virus surface antigen (HBsAg). This was done using a commercially available rapid test cassette (Amitech Diagnostics Inc.) kit. This is a one-step test for HBsAg in serum, plasma or whole blood. It utilizes the principle of immunochromatography, which is based on antigen capture or sandwich principle. This method uses monoclonal antibody conjugated to colloidal gold and polyclonal antibodies immobilized on a nitrocellulose strip in a thin line. This kit can detect HBsAg in serum in a concentration as low as $1.0 \mathrm{ng} / \mathrm{ml}$. 


\subsection{Data Analysis}

All statistical data and analyses was done using SPSS version 20. This was used to generate frequency tables and bivariate logistic regression was used to determine the association between socio-demographic factors and HBV seropositivity. Categorical variables (HBV positive and negative cases) were compared using Chi-square. The association of the variables with HBV infection was considered significant at a $\mathrm{P}$ value of $<0.05$.

\section{Results}

\subsection{Socio-Demographic Characteristics of Study Participants}

Socio demographic features among the inmate and donor population differed in distribution. Majority (76.2\%) of the subjects in the inmate population were aged between 20 - 40 years. Out of the 130 respondents $86.9 \%$ were male while $13.1 \%$ were female. Out of the total number of respondents in this population $81.5 \%$ had only attained a primary school level of education (Table 1).

Out of the 130 respondents in the donor population 108 (83.1\%) were aged between 20 - 40 years. Majority (95.4\%) were male while 6 (4.6\%) were female. In regard to education attained, 67 (51.4\%) had attained a primary school level of education while 11 (8.5\%) had not attended school (Table 2).

Table 1. Socio-demographic features of inmates.

\begin{tabular}{cccc}
\hline VARIABLES & CATEGORIES & N & $\%$ \\
\hline \multirow{2}{*}{ Age } & $<20$ & 4 & 3.1 \\
& $20-40$ & 99 & 76.2 \\
Sex & $>40$ & 27 & 20.8 \\
& Male & 113 & 86.9 \\
& Female & 17 & 13.1 \\
\hline \multirow{2}{*}{ Education } & No Education & 6 & 4.6 \\
& Primary & 106 & 81.5 \\
& Secondary & 14 & 10.8 \\
& Tertiary & 4 & 3.1 \\
\hline
\end{tabular}

Table 2. Socio-demographic features of blood donors.

\begin{tabular}{cccc}
\hline VARIABLES & CATEGORIES & $\mathrm{N}$ & $\%$ \\
\hline \multirow{2}{*}{ Age Groups } & $20-40$ & 12 & 9.2 \\
& $>40$ & 108 & 83.1 \\
Gender & Male & 10 & 7.7 \\
\hline \multirow{2}{*}{ Level of Education } & Female & 124 & 95.4 \\
& No Education & 6 & 4.6 \\
& Primary & 11 & 8.5 \\
& Secondary & 67 & 51.5 \\
& Tertiary & 39 & 30.0 \\
& & 13 & 10.0
\end{tabular}




\subsection{Seroprevalence of HBV in both in-Mates and Blood Donors}

There was a difference in HBV seroprevalence between the two study populations. Among the inmates study population, 7 (5.4\%) were HBV seropositive while among the blood donor study population 4 (3.1\%) were seropositive (Table 3).

\subsection{Socio-Demographic Factors and Their Association with HBV}

Among the blood donor population who were seropositive for HBV 3 (75\%) were aged between 20 - 40 while none ( $0 \%$ ) of those who were aged below 20 years of age were HBV seropositive. These results did not differ greatly with those of the inmate population where among the seropositive cases, 5 (71.4\%) were reported in the age group 20 - 40 years. Similarly none of the inmates aged below 20 years of age showed seropositivity to HBV. There was a statistically significant association between HBV seroprevalence and gender. More males than females were seropositive irrespective of the study population (Table 4).

Table 3. HBV seroprevalence among in mates and blood donors.

\begin{tabular}{ccccc}
\hline HBV Seropositivity & \multicolumn{2}{c}{ Donors } & \multicolumn{2}{c}{ Inmates } \\
\hline & Frequency & Percent (\%) & Frequency & Percent (\%) \\
\hline NEGATIVE & 126 & 96.9 & 123 & 94.6 \\
POSITIVE & 4 & 3.1 & 7 & 5.4 \\
Total & 130 & 100.0 & & 100.0 \\
Std. Error of Mean & 0.02 & & & 0.02 \\
Std. Deviation & 0.17 & & & 0.22 \\
Range & 1.00 & & 1.00
\end{tabular}

Table 4. Sero-positivity rates distributions among donors and inmates based on variables.

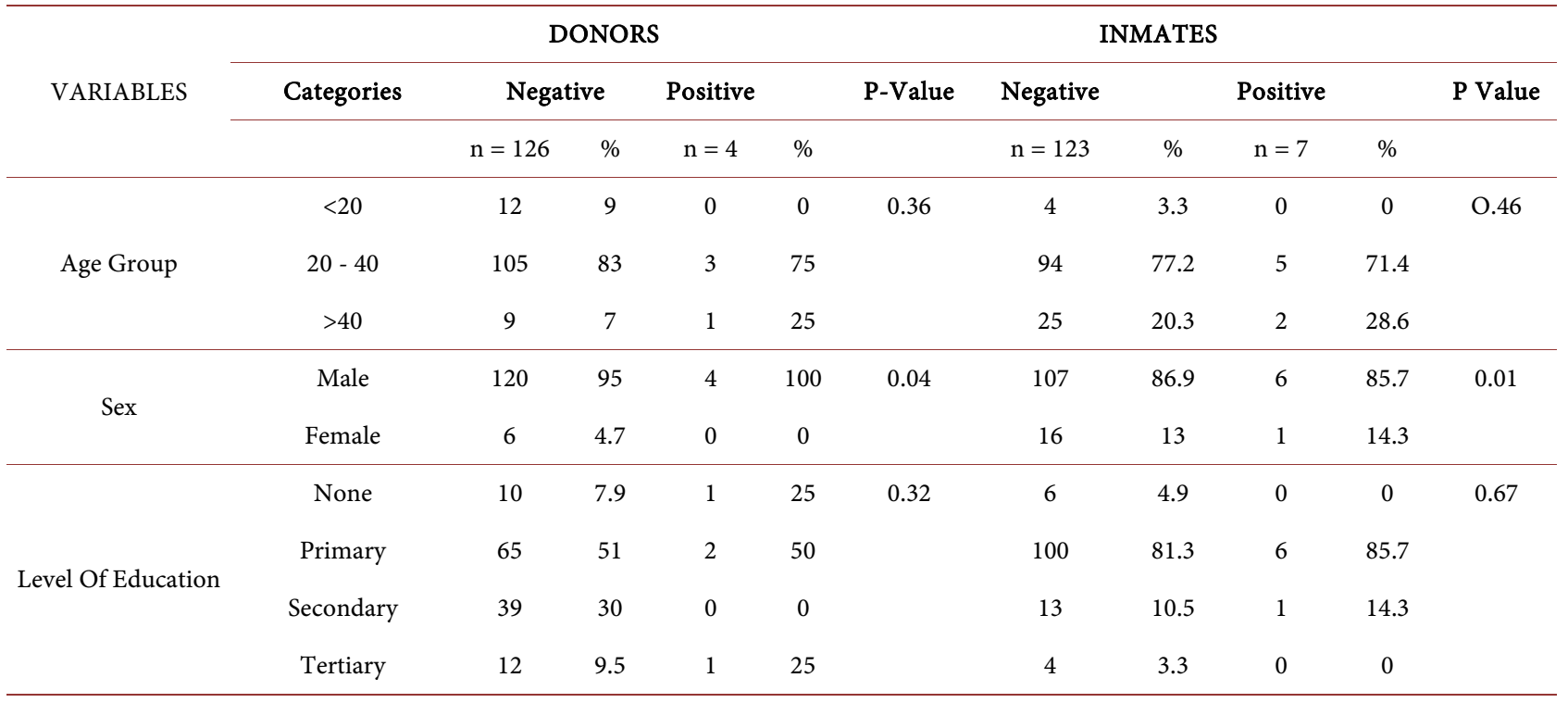




\section{Discussion}

Despite the availability of a safe and prophylactic vaccine, HBV continues to be a global public health problem. The prevalence of the Hepatitis B surface antigen (HBsAg), the hallmark of $\mathrm{HBV}$ infection varies significantly worldwide with sub Saharan Africa reporting the highest rates. Transmission of HBV infection is associated with certain risk factors. This can occur through exposure to body fluids and infectious blood enhanced via intravenous drug use and unprotected sex. Exposure can also occur at the time of birth [11]. Significant risk to transmission, has been identified through tattooing and acupuncture [12]. This puts recipients of blood transfusions and in-mates at a greater risk of HBV infection.

In the current study, the seroprevalence of HBV in the two study populations, differed significantly with in-mates having a higher seroprevalence than voluntary blood donors as shown in Table 3. These findings showed a higher seropositivity rate in the in-mates which are classified as high risk [13] compared to voluntary blood donors who are considered as low risk. This could be attributed to a higher risk of HBV transmission in prisons due to the lack of knowledge about HBV transmission modes [16]. Other risk factors associated with HBV transmission in prisons include frequent and unprotected sexual intercourse, injection drug use (IDU), tattooing and other forms of skin piercing [12] and trauma.

The HBV seroprevalence in voluntary blood donors was marginally higher compared to the $2.3 \%$ seroprevalence recorded in the same group in a study carried out in Nairobi, Kenya using the chemiluminescent microparticle immunoassay (CMIA) [8] and similar to (3.46\%) seroprevalence from a study carried out in Kisumu, Homabay and Siaya Counties, Kenya using ELISA and confirmed by CMIA [7].

These variations in HBV seroprevalence could be attributed to differences in geographical regions within the country as noted previously [6] and in differences in sensitivity of the screening methods used for the detection of HBsAg.

Consequently, the $5.4 \%$ seroprevalence of HBV from in-mates in this study was higher compared to the $3.3 \%$ recorded in a study among in-mates in Iran [17]. However, these findings are consistent with those from a study in Pakistan which showed HBV seropositivity of 5.6\% among prisoners and (3.1\%) in a control group that was comprised of blood donors [18]. These variations could still be attributed to differences in geographical locations and differences in the sensitivity of the testing kits used.

There was a significant association between HBV seropositivity and gender in the current study irrespective of the study group. More males were HBV seropositive compared to females in both study populations. A study in Pakistan on HBV infection among different sex and age groups, depicted the same trend of more males than females being infected with HBV [19]. Men are more exposed to risk factors associated with HBV infection than women especially use of intra-venous drugs and alcoholism that exposes them to unprotected sex com- 
pared to women. However, it is important to note that more males were sampled in this study than females in both populations. Majority (95.4\%) of the donors were male while only $6(4.6 \%)$ were females compared to $(86.9 \%)$ male and (13.1\%) female in the in-mate population (Table 1 and Table 2).

Even though, there was no significant association of HBV seropositivity with age among both in-mates and donors, there were differences in seropositivity observed. Age was stratified into three sets $(<20,20-40$ and $>40)$ years. The middle age set recorded majority of the respondents.

Out of the 130 respondents in the donor population, 108 (83.1\%) were aged between 20 - 40 years of age compared to in-mates where $76.2 \%$ were aged between 20 - 40 years. Comparing HBV seropositivity in both study populations in this particular age set, $3(75 \%)$ out of 4 blood donors and $5(71.4 \%)$ out of 7 in-mates were seropositive (Table 3 ). This therefore, translated to higher seropositivity rate in the 20 - 40 years age group compared to other two age groups which had lower HBV seropositivity rates in both populations. This could be attributed to much social activity and experimental behavior harbored by the youths in this age group. Consequently, there is a risk of exposure to the HBV risk factors from the said behaviors. However, the other two age groups with lower seropositivity also had fewer study participants. Maybe any further increase on their recruitment could have altered the results.

\section{Conclusions}

In conclusion, the study findings depicted HBV seroprevalence of 5.4\% from the in-mates and $3.1 \%$ in voluntary blood donors. Consistent with other studies that show prison in mates as HBV high risk populations, this study has shown a significantly higher seroprevalence of HBV compared to low risk voluntary blood donors. This is because in-mates are more exposed to HBV infection risk factors than the general population.

Additionally, $100 \%$ seropositivity for donors and $85.7 \%$ for the in-mates were males. Males therefore had a significantly higher HBV seroprevalence compared with females irrespective of the study group. Men tend to get more exposed to $\mathrm{HBV}$ infection risk factors due to their more active behavior compared to women.

Age of the participants in both study populations influenced results to a certain degree. Out of the 130 respondents in the donor population, $83.1 \%$ were aged between 20 - 40 years of age compared to in-mates where $76.2 \%$ were aged between 20 - 40 years. Comparing HBV seropositivity in both study populations in this particular age set, $75 \%$ of blood donors and $71.4 \%$ of in-mates were seropositive. This could be attributed to much social activity and experimental behavior harbored by the youths in this age group.

These findings particularly among prisoners, can serve as a proxy for other prisons within the country due to similarities shared in the set-ups since no data exists regarding $\mathrm{HBV}$ infection in Kenyan prisons. Further studies to determine 
other predisposing risk factors should be conducted in the study area. Moreover, molecular studies to determine circulating HBV genotypes in this group of people and region are required.

\section{Acknowledgements}

All authors would like to thank the study participants for their willingness to participate.

\section{Funding}

Funding of this study was done by the individual authors.

\section{Conflicts of Interest}

The authors declare no conflicts of interest regarding the publication of this paper.

\section{References}

[1] World Health Organization (WHO) (2018) What Is Hepatitis. World Health Organization, Geneva.

[2] Hutin, Y., Nasrullah, M., Easterbrook, P., Nguimfack, B.D., Burrone, E., Averhoff, F., et al. (2018) Access to Treatment for Hepatitis B Virus Infection-Worldwide, 2016. Morbidity and Mortality Weekly Report, 67, 773-777. https://doi.org/10.15585/mmwr.mm6728a2

[3] Cui, F., Shen, L., Wang, H., Wang, F., Bi, S., Liu, J., et al. (2017) Prevention of Chronic Hepatitis B after 3 Decades of Escalating Vaccination Policy, China. Emerging Infectious Diseases, 23, 765-772. https://doi.org/10.3201/eid2305.161477

[4] Breakwell, L., Tevi-Benissan, C., Childs, L., Mihigo, R. and Tohme, R. (2017) The Status of Hepatitis B Control in the African Region. The Pan African Medical Journal, 27, Article No. 17. https://doi.org/10.11604/pamj.supp.2017.27.3.11981

[5] Zampino, R., Boemio, A., Sagnelli, C., Alessio, L., Adinolfi, L.E., Sagnelli, E., et al. (2015) Hepatitis B Virus Burden in Developing Countries. World Journal of Gastroenterology, 21, 11941-11953. https://doi.org/10.3748/wjg.v21.i42.11941

[6] Mutuma, G., Mbuchi, M.W., Zeyhle, E., Fasana, R., Okoth, F.A., Maina Kabanga, J., et al. (2011) Prevalence of Hepatitis B Virus (HBV) Surface Antigen and HBV-Associated Hepatocellular Carcinoma in Kenyans of Various Ages. African Journal of Health Sciences, 18, 53-61.

[7] Onyango, C.G., Ogonda, L., Guyah, B., Okoth, P., Shiluli, C., Humwa, F., et al. (2018) Correction to: Seroprevalence and Determinants of Transfusion Transmissible Infections among Voluntary Blood Donors in Homabay, Kisumu and Siaya Counties in Western Kenya. BMC Research Notes, 11, Article No. 410.

https://doi.org/10.1186/s13104-018-3521-4

[8] Aluora, P.O., Muturi, M.W. and Gachara, G. (2020) Seroprevalence and Genotypic Characterization of HBV among Low Risk Voluntary Blood Donors in Nairobi, Kenya. Virology Journal, 17, Article No. 176. https://doi.org/10.1186/s12985-020-01447-2

[9] Karra, V.K., Chowdhury, S.J., Ruttala, R., Polipalli, S.K. and Kar, P. (2016) Clinical Significance of Quantitative HBsAg Titres and its Correlation With HBV DNA Le- 
vels in the Natural History of Hepatitis B Virus Infection. Journal of Clinical and Experimental Hepatology, 6, 209-215. https://doi.org/10.1016/j.jceh.2016.07.002

[10] Hadziyannis, E. and Laras, A. (2018) Viral Biomarkers in Chronic HBEAG Negative HBV Infection. Genes, 9, Article No. 469. https://doi.org/10.3390/genes9100469

[11] Miri, S.M. and Alavian, S.M. (2011) Risk Factors of Hepatitis B Infection: Health Policy Makers Should Be Aware of Their Importance in Each Community. Hepatitis Monthly, 11, 238-239.

[12] Jafari, S., Copes, R., Baharlou, S., Etminan, M. and Buxton, J. (2010) Tattooing and the Risk of Transmission of Hepatitis C: A Systematic Review and Meta-Analysis. International Journal of Infectious Diseases, 14, E928-E940.

https://doi.org/10.1016/j.ijid.2010.03.019

[13] Ramamoorthy, M., Venketeswaran, A., Seenivasan, P., Revathy, M., Manimaran, M., Chitra, S., et al. (2016) Risk Factors and Prevalence, Hepatitis B Virus and Hepatitis C Virus among Prison Inmates, Chennai, India, 2015. International Journal of Infectious Diseases, 53, Article No. 90. https://doi.org/10.1016/j.ijid.2016.11.227

[14] Ahmed Abade, M. (2012) Prevalence and Risk Factors for Hepatitis B Infection among Pregnant Women Attending Antenatal Clinics in Garissa District. 1-19.

[15] Casagrande, J.T., Pike, M.C. and Smith, P.G. (1978) An Improved Approximate Formula for Calculating Sample Sizes for Comparing Two Binomial Distributions. Biometrics, 34, 483-486. https://doi.org/10.2307/2530613

[16] Gétaz, L., Casillas, A., Siegrist, C.A., Chappuis, F., Togni, G., Tran, N.T., et al. (2018) Hepatitis B Prevalence, Risk Factors, Infection Awareness and Disease Knowledge among Inmates: A Cross-Sectional Study in Switzerland's Largest Pre-Trial Prison. Journal of Global Health, 8, Article ID: 020407. https://doi.org/10.7189/jogh.08.020407

[17] Zary Nokhodian, M.Y. (2014) Seroprevalence of Hepatitis B Markers among Incarcerated Intravenous Drug Users.

[18] Fayyaz, M. (2006) Frequency of Hepatitis B and C Seropositivity in Prisoners. Biomedica, 22, 55-58.

[19] Khan, F., Shams, S., Qureshi, I.D., Israr, M., Khan, H., Sarwar, M.T., et al. (2011) Hepatitis B Virus Infection among Different Sex and Age Groups in Pakistani Punjab. Virology Journal, 8, Article No. 225. https://doi.org/10.1186/1743-422X-8-225 